\title{
«Le Symboliste», une revue littéraire de courte vie mais de grande importance
}

\section{Veronica Gatti}

\section{(2) OpenEdition}

1 Journals

\section{Édition électronique}

URL : https://journals.openedition.org/studifrancesi/2958

DOI : 10.4000/studifrancesi.2958

ISSN : 2421-5856

Éditeur

Rosenberg \& Sellier

\section{Édition imprimée}

Date de publication : 1 juillet 2013

Pagination : 369-381

ISSN : 0039-2944

\section{Référence électronique}

Veronica Gatti, « «Le Symboliste», une revue littéraire de courte vie mais de grande importance », Studi Francesi [En ligne], 170 (LVII | II) | 2013, mis en ligne le 30 novembre 2015, consulté le 31 janvier 2023. URL : http://journals.openedition.org/studifrancesi/2958; DOI : https://doi.org/10.4000/studifrancesi. 2958

\section{cc) (†) $\odot$}

Creative Commons - Attribution - Pas d'Utilisation Commerciale - Pas de Modification 4.0 International - CC BY-NC-ND 4.0

https://creativecommons.org/licenses/by-nc-nd/4.0/ 


\title{
"Le Symboliste», une revue littéraire de courte vie mais de grande importance
}

\begin{abstract}
The first issue of the literary weekly «Le Symboliste» came out on October $7^{\text {th }} 1886$. The journal ceased publication, after only four issues, on October $30^{\text {th }}$ that same year.

It was founded by Gustave Kahn, managing editor, Paul Adam, editorial secretary, and Jean Moréas, editor-in-chief. Many writers and intellectuals contributed to the lively debate contained in its pages.

The aim of the following article is to point out the importance the magazine had in the literary revolution of Symbolism by offering a detailed analysis of the contributions made by all those who worked on the journal. This analysis helps us understand the construction process of the Symbolist movement aesthetics and appreciate the varied character it showed from the very start.
\end{abstract}

Il 7 ottobre 1886 viene pubblicato il primo numero del settimanale letterario «Le Symboliste», che uscirà in soli quattro numeri fino al 30 ottobre 1886. La rivista è creata da Gustave Kahn, direttore, Paul Adam, segretario di redazione e da Jean Moréas nel ruolo di redattore capo; ma diversi scrittori e intellettuali animano il dibattito all'interno delle pagine.

Il presente articolo si propone di evidenziare l'importanza che questa rivista ha avuto nello sviluppo della rivoluzione letteraria del Simbolismo, attraverso l'analisi approfondita dei contributi di tutti i suoi collaboratori. Tale analisi ci consente di comprendere il processo di costruzione dell'estetica del movimento simbolista e inoltre di coglierne il carattere eterogeneo già presente fin dai suoi esordi.

La revue littéraire «Le Symboliste» a souvent été l'objet d'analyses assez génériques au sujet des revues littéraires qui ont marqué la fin du XIX ${ }^{e}$ siècle: Michael James Wroblewski en 1978 a publié Four symbolists periodicals. Toward the definition of an esthetic, il s'agit d'une étude qui prend en considération quatre revues littéraires, notamment la «Revue Wagnérienne», «La Revue Indépendante», «La Vogue» et «Le Symboliste» ${ }^{1}$. Plus récemment Yoan Vérilhac a produit un autre texte assez générique qui, tout en prenant en considération les petites revues de l'époque symboliste, ne rentre pas dans les détails de l'analyse, mais se contente de suggérer une vue d'ensemble 2 .

D'autres spécialistes n'ont repris de cette revue que les articles, auteur par auteur, qui faisaient l'objet de leur recherche: par exemple Mireille Dottin, comme nous le verrons, reprend un texte de critique d'art que Jules Laforgue publie dans «Le Symboliste» pour l'intégrer au volume Textes de critique d'art de Laforgue dont elle soigne la présentation.

(1) M.J. Wroblewski, Four symbolists periodicals. Toward the definition of an esthetic, dans «Dissertation Abstracts International» vol. 38, 8 Février 1978.
(2) Y. VÉRILHAC, La jeune critique des petites revues symbolistes, Saint-Etienne, Presses univ. de StEtienne, 2010, p. 339. 
Ce qui nous intéresse à présent est donc de mettre en évidence l'importance que cette petite revue a eu dans le développement de l'école symboliste: une analyse approfondie des contributions de tous les collaborateurs permet de comprendre le processus de construction de l'esthétique du mouvement symboliste et encore plus de cueillir le caractère hétérogène que ce mouvement présente depuis sa naissance.

Le 7 octobre 1886 le premier numéro de l'hebdomadaire littéraire «Le Symboliste» fait sa parution. Il est créé par Gustave Kahn, directeur, Paul Adam, secrétaire de la rédaction et surtout par celui que nous pouvons considérer comme son véritable moteur, Jean Moréas, dans le rôle de rédacteur en chef. Plusieurs écrivains et intellectuels de l'époque collaborent à la revue et l'enrichissent d'articles et d'opinions qui nous aident à deviner le climat de fervent débat littéraire qu'on devait vivre en ce moment.

Cette revue aura une vie très courte: en effet, son dernier numéro date du 30 octobre de la même année; elle compte, en total, seulement quatre numéros, mais elle sera d'importance vitale pour le développement du Symbolisme, permettant la prise de conscience nécessaire qui aboutira à celle que nous pouvons définir comme une révolution littéraire.

«Le Symboliste» n'est pas la première revue qui se fait le porte parole de la poétique des nouvelles générations: en effet, plusieurs revues étaient déjà surgies sous l'impulsion de groupes de l'avant-garde littéraire. Leur histoire est d'ailleurs bien connue $^{3}$, mais c'est au cours de 1886 que notre révolution arrive à son apothéose.

On ne peut pas se passer de rappeler qu'au printemps 1886 une floraison de petites revues porte au découvert le débat littéraire en cours: au mois d'avril nous assistons à la naissance de la revue «Le Décadent» sous la direction d'Anatole Baju et de «La Vogue» dirigée par Léo d'Orfer et Gustave Khan.

Après la publication du Manifeste du Symbolisme de Moréas, dans «Le Figaro» du 18 septembre 1886, la situation semble devenir de plus en plus complexe: les groupes et les artistes s'affrontent et rivalisent pour défendre leurs positions en choisissant les petites revues littéraires comme champ de bataille.

Des querelles naissent aussi au sein du Symbolisme: René Ghil en réclame la paternité en considérant son Traité du Verbe comme la théorisation de la nouvelle

(3) Nous pouvons citer à titre d'exemple les plus significatives: «L'Hydropathe», né en 1879, qui va prendre le nom «Tout-Paris» dans ses derniers mois de vie en 1880; «Le Chat Noir», organe du groupe homonyme; «La Nouvelle Rive Gauche» qu'en 1883 prendra le nom de «Lutèce».

En 1884 on assiste aussi à la naissance de «La Revue Indépendante» qui est encore assez liée à la littérature traditionnelle, mais elle exprime son admiration pour Richard Wagner qui vient de mourir (en 1883) et qui commence à être reconnu comme un véritable novateur.

Suite à l'intérêt qui s'est créé autour de Wagner, Edouard Dujardin décide de fonder la «Revue Wagnérienne», dont le premier numéro paraît le 8 février 1885 .

Le $1^{\text {er }}$ décembre 1885 on publie le premier numéro de la revue «Le Scapin», dirigé par E. Raymond, et en mars 1886 voit le jour le premier numéro de la revue «La Pléiade», dirigée par Rodolphe Darzens.

Il ne faut pas oublier que l'avant-garde littéraire est très active aussi en Belgique: en novembre 1879 Verhaeren, Deman, Gilkin et d'autres jeunes in- tellectuels fondent «La Semaine des Etudiants», un journal qui s'intéresse à la littérature, mais qui critique aussi la société contemporaine et la classe bourgeoise.

En 1880 Max Waller publie «Le Type», qui rivalise avec «La Semaine des Etudiants»; à cause des conflits que ces deux journaux produisent, l'université ordonne d'en suspendre la publication.

L'année suivante Picard fonde la revue «L'Art Moderne» et Max Waller la «Jeune Belgique», ces revues donnent leur contribution à la diffusion du nouveau goût poétique et artistique qui semble se répandre très rapidement aussi en Belgique.

En 1884 Ricard et Lemonnier fondent la revue «Société Nouvelle»; presque en même temps Albert Mockel, âgé de dix-huit ans seulement, fonde à Liège la revue «L'Élan littéraire», qui, en 1886 , change son nom et devient «La Wallonie». «La Wallonie» est parmi les revues les plus importantes de la période symboliste.

Pour finir nous pouvons citer encore $\ll \mathrm{La} \mathrm{Ba}$ soche», revue belge qui publie une série d'articles théoriques de Ghil qui peuvent être considérés comme la première version de son Traité du Verbe. 
poésie; Jean Moréas fait de même, il se considère comme le premier véritable théoricien du mouvement puisque il a rédigé et donné à la presse le Manifeste de la nouvelle école.

Entre-temps E. Raymond, directeur du «Scapin», décide de fonder un nouveau journal: «La Décadence» et il en propose la direction à René Ghil ${ }^{4}$.

Ghil accepte la proposition et il demande la participation de Mallarmé. Dans le premier numéro, qui paraît le $1^{\text {er }}$ octobre 1886 , il profite pour renouveler son concept d'art poétique élaboré dans le Traité du Verbe et surtout pour attaquer les adversaires.

Dans son article Notre École, il précise que «trop longtemps, sous le titre générique le Décadent [...] trop d'adverses aspirations se sont gênées [...] Deux maîtres amis, opposés de vues et de manière, rêvent sur le mouvement qu'ils engendrent: ce sont Mallarmé et Verlaine» et il accuse les Décadents de vouloir «se parer de l'honneur du Symbole» dont la doctrine a été «longuement exposée au Traité du Verbe»5.

La réponse de Moréas à ces attaques ne tarde pas et le 7 octobre 1886 le premier numéro de la revue littéraire «Le Symboliste» est publié.

\section{La structure de la revue}

«Le Symboliste» se constitue de quatre pages par numéro, la première sortie porte le sous-titre «Journal hebdomadaire paraissant le jeudi»: ce sous-titre, dans les publications suivantes, change en «Journal littéraire et politique paraissant le jeudi». Toutefois, si nous considérons les dates sur chaque numéro, il est évident que le jour de sortie n'a pas toujours été respecté: en effet, le premier numéro va du 7 au 14 octobre 1886, le deuxième du 15 au 22 octobre, le troisième du 22 au 29 octobre et le quatrième du 30 octobre au 6 novembre.

Chaque numéro est complété par un petit sommaire qui introduit au lecteur les titres des articles et les auteurs respectifs et par l'avis de la rédaction qui donne une description des rubriques du journal et fournit les noms de tous les collaborateurs ${ }^{6}$. L'avis de la rédaction est normalement positionné à la suite des deux premiers articles, sauf dans la première sortie où il se trouve entre le premier et le deuxième.

\section{Jean Moréas}

Jean Moréas, comme nous l'avons précisé auparavant, peut être considéré comme le véritable moteur de cette petite revue et sa collaboration compte de trois articles, dont les deux premiers, Chronique et Une réponse, publiés dans «Le Symboliste» du 7 octobre 1886, se chargent de préciser le caractère de la nouvelle école littéraire et ressemblent à une sorte de prolongement du Manifeste, tandis que le troisième, Peintures, publié dans le numéro du 22 octobre, n'est qu'une présentation de l'œuvre de Félix Fénéon Les Impressionnistes en 1886, dont Moréas parait partager

(4) G. Michaud, Message poétique du Symbolisme, Paris, Librairie Nizet, t. II, 1947 p. 346. Il faut préciser que E. Raymond sera le directeur de «La Décadence» tandis que René Ghil aura le rôle de rédacteur en chef.

(5) G. Michaud, Message poétique du Symbolisme, cit., t. II, p. 346.

(6) Les collaborateurs cités dans l'avis de la rédaction sont: Charles Henry, Felix-Fénéon, Jules
Laforgue, Jean Ajalbert, Gaston Dubreuilh, Maurice Barrès, Edouard Dujardin, J.-K. Huysmans, Stéphane Mallarmé, Francis Poictevin, Paul Verlaine, Charles Vignier, Teodor de Wyzewa, auxquels s'ajoutent Paul Margueritte et Camille de Sainte-Croix dans les deux dernières publications. Malheureusement quelques uns entre eux ne signeront aucun article dans le journal. 
tous les avis et, en allant plus loin encore, il acclame Puvis de Chavannes comme celui qui entraîne la peinture aussi vers le langage du symbole. En ce qui concerne le style de l'article, Moréas reste fidèle à lui-même, il est toujours sophistiqué dans le choix du langage, il utilise des mots inusuels et anciens, souvent empruntés à Rabelais ${ }^{8}$.

Voyons donc de plus près ses deux premiers articles.

Le premier numéro du «Symboliste» s'ouvre par l'article Chronique signé par Moréas. Cet article est organisé sous forme de dialogue dans lequel l'auteur fait jouer deux personnages: Fortunato et Vondervotteimittis. Le dialogue est, en quelque sorte, un escamotage que Moréas utilise pour préciser une nouvelle fois le concept de subjectivité de l'art symboliste qu'il avait déjà exprimé dans son Manifeste où il déclarait: «le roman symbolique édifiera son œuvre de déformation subjective, fort de cet axiome: que l'art ne saurait chercher en l'objectif qu'un simple point de départ extrêmement succinct».

Comme d'habitude Moréas revendique l'importance d'un langage riche et suggestif, pour cette raison il fait ouvrir le dialogue à Fortunato par la description d'un lieu en employant des mots très inusuels ${ }^{9}$. Le deuxième interlocuteur, Vondervotteimittiss, probablement étonné par cette description, demande à Fortunato: «de quel pays de fables voulez-vous parler?», Fortunato répond: «Du boulevard des Italiens», alors Vondervotteimittis commente: «Mais votre peinture est fausse de tout point» et Fortunato réplique: «l'objectif n'est que pur semblant, qu'apparence vaine qu'il dépend de moi de varier, de transmuer et d'anéantir à mon gré» ${ }^{10}$.

(7) Moréas reprend des extraits du texte de Fénéon: tout d'abord une petite introduction où on précise que le public a toujours reconnu à Manet le rôle principale dans la naissance de l'Impressionnisme mais, en réalité, Camille Pissarro, Degas, Renoir et Monet «furent les chefs de la révolution», tandis que Manet en fut tout simplement le «héraut». Ensuite on passe à analyser le travail de Degas, Federico Zandomeneghi, Gauguin, Armand Guillaumin, David Estoppey, Jean-François Raffaëlli, Claude Monet. Pour terminer Moréas reprend directement la parole: il exprime son admiration pour Puvis de Chavannes et ensuite il consacre un éloge à Félix Fénéon pour le choix stylistique et lexical qui donne un magnifique caractère à son œuvre et qui fait de lui un «merveilleux écrivain».

(8) Dans son introduction Moréas affirme que «le hourt» entre le goût classique dominant et les nouveaux peintres «fut impétueux»; ainsi il rédige une liste de sujets classiques avec un ton assez dénigrant, empruntant beaucoup de définitions au langage de Rabelais comme par exemple: «Bibliques pauvresses à la chair atramentée, Abrahams tintalorisés, Jésus à barboire de droguiste, cardinaux concilipètes, martyrs fustés et hagards, mercenaires caterves, "mammalement scandaleuses" Madones, Titans halbrenés, Vénus napleuses, Nymphes tébides, Napoléons de banlieues».

Plus loin il explique que, suite à la confrontation entre la peinture traditionnelle et les peintres innovateurs, les «carêmes-prenants du quai Malaquais» ou, autrement dit, les peintres ridicules de l'École des beaux-arts située au quai Malaquais, tous «croustelevés de bleu de Prusse, de bitume et de terre de Sienne» ont quitté leurs «chevalets et les cadres» et, envahis par une sorte de «terreur panique», se sont enfuis et ils ont laissé la place au nouveaux peintres qui se sont finalement imposés malgré les adversités représentés par «les bêlements niais du publique» et aussi par «les imprécations des morosophes clavés au lieu commun».

Moréas ajoute qu'Emile Zola et Théodore Duret se sont chargés de relater cette bataille et le «ponocrate» Huysmans pour célébrer la nouvelle peinture a entonné «des dithyrambes abasourdis».

(9) En 1889, une fois que le Symbolisme s'était affirmé, Léon Vanier demande à Moréas l'autorisation de publier des articles parus en 1885-1886, en pleine période de révolution symboliste, avec l'intention de les réunir en une brochure qui portera le titre Les premières armes du Symbolisme. Moréas lui écrit une lettre en réponse où il fait allusion à des souvenirs de l'époque. Entre autre, il avoue avoir parfois laissé ses mots dépasser sa pensée, surtout dans l'article Une réponse adressé à Anatole France, paru aussi dans «Le Symboliste» du 7 octobre 1886, où il affirmait: «J'admire Baudelaire tout en estimant Lamartine»; mais, en échange de cet aveu, il demande indirectement à France de lui «faire grâce», à son tour, des mots «torcol et bardocucule, deux bons vieux mots que j'ai employés quelque part et qui l'irritent». C'est justement dans la description qui ouvre l'article Chronique qu'il avait employé la définitions «bardocuculés» tandis que le terme «torcol» se retrouve dans Les Demoiselles Goubert: moeurs de Paris. Dans sa lettre à Vanier, Moréas ajoute aussi l'explication de ces deux mots «torcol est net et bien formé, quant à bardocucule, il signifie la mante à capuchon des anciens Gaulois».

Cfr. J. Plowert, Petit Glossaire pour servir à l'intelligence des auteurs décadents et symbolistes, Léon Vannier, Paris, 1888

(10) Ce qui est reproduit en italique respecte les caractères du texte original. Chronique, «Le Symboliste», 7 octobre 1886 . 
Ce qui nous semble intéressant de souligner c'est le choix du nom des personnages protagonistes de l'article Chronique: en effet, Moréas reprend ces noms de deux contes d'Edgar Allan Poe qui font partie du volume Nouvelles Histoires fantastiques, traduit par Baudelaire et publié en 1857.

Le nom de Vondervotteimittiss est repris du conte Le diable dans le beffroi de $1839^{11}$, tandis que le nom de Fortunato est emprunté au récit La barrique d'amontillado de $1846^{12}$. Quelles sont les raisons de ces choix? Moréas semble par là vouloir établir un rapport entre l'élément perturbateur du diable dans le beffroi et l'effet perturbateur qui a eu le Symbolisme sur la critique et la presse officielle qui n'arrêtent pas de le persécuter. De même, le personnage de Fortunato, en dépit de son nom, fait allusion à un pauvre infortuné, persécuté sans raison, comme il arrive au Symbolisme naissant, dont la seule faute est de ne pas être compris par les «esprits paresseux et routiniers» qui «aiment à entendre aujourd'hui ce qu'ils entendaient hier»; pour utiliser une expression que Moréas avait empruntée à Alfred de Vigny et qu'il avait employée dans son article publié le 11 août 1885 dans le «XIX Siècle».

Moréas poursuit ainsi dans le style de son Manifeste, puisque dans les deux cas il a recours à la forme du dialogue entre personnages. Ici il met en scène l'échange entre Fortunato et Vondervotteimittis, tandis que dans le Manifeste littéraire il propose un petit Intermède consistant en un dialogue entre trois personnages: un détracteur de l'école symbolique, Théodore de Banville et Erato $^{13}$.

Dans ce premier numéro du «Symboliste» Moréas signe aussi l'article Une réponse: il s'agit d'une lettre en réponse à l'article d'Anatole France paru dans «Le Temps» du 26 septembre 1886 qui était, à son tour, une critique au Manifeste littéraire du «Figaro».

Il est évident, d'après le débat auquel on assiste dans la presse de l'époque, que les nouveautés apportées par les Symbolistes causent beaucoup d'étonnement parmi les critiques qui, de leur côté, ne perdent pas l'occasion pour exprimer des jugements assez sévères.

Les attaques principales contre les poètes symbolistes concernent le choix volontaire d'un langage obscur qui prétend retrouver ses racines dans un passé lointain et d'un style qui se propose de rompre tout rapport avec la métrique classique, typique de l'époque et acclamée par les académiciens.

Dans cette lettre Moréas assume un ton polémique pour contester ces reproches et défendre sa position: le Symbolisme se propose de couper les ponts avec la littérature contemporaine qui, excepté quelques tentatives de renouvellement mal abouties, se présente comme vide de contenus et sans aucune originalité ${ }^{14}$. Moréas revendique

(11) Le diable dans le beffroi narre l'histoire imaginaire du petit bourg hollandais de Vondervotteimittiss où tout était absolument parfait jusqu'à l'arrivée d'un élément nouveau qui vient perturber l'ordre et l'équilibre: le perturbateur est un jeune homme qui, dès son arrivée, s'empare du clocher de l'église déréglant l'heure et la vie du bourg. Le narrateur de l'histoire fait «appel à tous les amants de l'heure exacte» et il les incite à marcher «en masse sur le bourg» pour restaurer «l'ancien ordre des choses [...] en précipitant ce petit drôle du clocher».

(12) La barrique d'amontillado raconte la fin cruelle de Fortunato, un jeune italien qui rencontre un soi-disant ami pendant qu'il est en train de fêter le carnaval; l'ami, sous le prétexte de lui faire goûter du vin qu'il vient d'acheter, l'amène dans le sous-sol de sa villa et l'enferme dans un mur, provoquant ainsi sa mort.

(13) www.gallica.fr, J. MORÉAs, Les premières armes du Symbolisme, Léon Vanier, Paris, 1889, p. 35.

(14) Selon Moréas il y a eu deux tentatives d'apporter un renouveau: le Romantisme et le Naturalisme. Premièrement le Romantisme a donné l'impression de vouloir engager une révolution, mais il n'a pas eu le courage d'aller jusqu'au bout. Pour cette raison, dans son Manifeste Moréas met en scène un petit Intermède où il confie la défense de la nouvelle poésie à Théodore de Banville, poète romantique, mais en lui faisant avouer l'échec de sa mission. Pour ce qui concerne le Naturalisme, Moréas pense qu'il a été simplement une «protestation légitime, mais mal avisée, contre les fadeurs de quelques romanciers alors à la mode». 
le retour à la langue du XVI siècle (la même de l'historien Philippe de Commines et du maittre François Rabelais), la liberté de versification et surtout le principe de la subjectivité, complètement effacée par plus de deux siècles de rationalisme et de réalisme.

Pour finir, Moréas identifie la possible origine de ses controverses avec Anatole France dans le fait que ce dernier admire «Lamartine, tout en estimant Charles Baudelaire», tandis que lui, au contraire, il «admire Baudelaire tout en estimant Lamartine» ${ }^{15}$.

\section{Paul Adam}

Paul Adam, dans «Le Symboliste», signe deux articles qui paraissent dans les mêmes numéros que ceux signés par Jean Moréas ${ }^{16}$. Les deux auteurs semblent avoir une bonne entente, confirmée par leur collaboration aux œuvres Les Demoiselles Goubert: mours de Paris et Le Thé chez Miranda ${ }^{17}$.

La Presse et le Symbolisme est le premier des deux articles d'Adam et il parait dans «Le Symboliste» du 7 octobre 1886.

Dans ces deux colonnes l'auteur cherche à donner une définition de l'art symboliste qui est en train de créer sa place à l'intérieur du panorama littéraire, mais qui est encore forcée de faire face aux hostilités de la critique officielle et de la presse.

Tout d'abord Adam se préoccupe de faire la distinction entre le Symbolisme et le Décadentisme. Il considère le Décadentisme comme un mouvement né sur un malentendu, causé par la publication de l'œuvre parodique de Vicaire et Beauclair ${ }^{18}$, et sur une théorie mal étoffée, présentée par Baju dans la revue «Le Décadent». Ả son avis c'est la presse qui a inventé le phénomène, en amplifiant un courant qui fort probablement n'aurait d'autre façon qu'un poids insignifiant dans l'histoire littéraire. Il retrace ensuite à son tour l'histoire du Symbolisme et il met en relief le principe de la subjectivité de l'art, en proposant ses justifications: une société évoluée nécessite d'une littérature prête à fournir une multitude de sensations à son public et la poésie symboliste semble répondre parfaitement à cette exigence. Tout comme Moréas il identifie le Symbolisme avec la recherche d'un langage poétique qui se traduit directement en sensations et images, grâce à la synthèse d'un mot évocatoire et d'un rythme capable de créer de puissantes suggestions. Pourtant il réintroduit le principe rhétorique traditionnel de la différence des styles en fonction des sujets traités: il admet un langage assez simple, utilisé pour des buts purement descriptifs, destiné à un public assez vaste, et un langage d'ordre supérieur, celui du symbole, adapté à des thèmes plus spirituels, compréhensible à un public restreint ${ }^{19}$. Dans ce cas il partage la volonté de Moréas de se ressourcer à la langue du Xvi siècle, et notamment de Rabelais, en s'écartant complètement des deux siècles successifs qui n'ont produit qu'une littérature médiocre et vraiment décadente ${ }^{20}$. Le poète qu'Adam envisage est enfermé dans sa tour d'ivoire, complètement absorbé par son art, s'écartant volontairement de la foule vulgaire. Le mouvement de séparation entre l'art et la vie, com-

(15) Dans la brochure Les premières armes $d u$ Symbolisme, comme nous l'avons dit, Moréas tient à rectifier cette phrase, il admet avoir peut-être exagéré avec les mots puisque, en vérité, il a toujours admiré Lamartine autant que Baudelaire.

(16) Plus exactement dans le premier et dans le troisième numéro.

(17) Les deux œuvres sont éditées par Tresse et Stock, Paris, 1886.
(18) Les Déliquescences, poèmes décadents d'Adoré Floupette, 1885.

(19) Adam est le seul, parmi les collaborateurs de la revue, qui revendique ouvertement la possibilité d'employer l'usage de deux styles différents selon le sujet traité.

(20) Nous pouvons remarquer à ce propos une forte similitude avec les idées exprimées par Moréas. 
mencé par le Parnasse et radicalisé par Mallarmé, est donc ici considéré comme une évolution naturelle et inévitable.

Dans le troisième numéro du «Symboliste» paru le 22 octobre, Adam signe l'article Chronique, un morceau artistique, où l'auteur s'amuse à donner un exemple de son style poétique. Il emploie des mots puissants, recherchés et choisis avec soin extrême, capables d'évoquer au lecteur des images mentales riches en détails et en sensations très vives.

Il s'agit d'une longue série de croquis de vie parisienne qui peignent différentes situations de l'existence quotidienne vécue à l'intérieur des habitations, dans les rues, dans les cafés de la ville ou encore dans la salle d'un tribunal. La vision qu'ils offrent est tout à fait subjective, au moyen aussi de jeux de lumière. Telle est la présentation de la salle des Assises au tomber de la nuit: les objets, y compris le crucifix des serments, subissent l'effet de distorsion causé par l'éclairage des bougies.

\section{Gustave Kabn}

Ce qui frappe tout de suite le lecteur attentif est que les articles de Moréas et Adam n'apparaissent jamais dans un même numéro que ceux de Kahn ${ }^{21}$.

Il parât que ceci n'est pas dû à une simple coïncidence, mais plutôt au fait qu'entre Kahn et Moréas il y avait des incompréhensions concernant les théories symbolistes $^{22}$, tandis qu'Adam et Moréas étaient liés par beaucoup de points communs. Kahn est évidemment plus politique, il a une vision différente du rapport entre le poète et la société, il paraît prendre ses distances de la tour d'ivoire.

Dans «Le Symboliste» du 15 octobre 1886, Kahn signe un article qui porte le titre Difficulté de vivre: c'est une «longue dissertation sur la médiocrité de l'existence dans la société bourgeoise» de son époque tout en gardant l'espoir d'un lendemain meilleur ${ }^{23}$.

Kahn brosse une esquisse de la société morne et sans espoir, telle qu'elle était en fait présentée déjà au début du Romantisme, mais qui correspond aussi à la vision fin de siècle. Par rapport aux autres symbolistes il s'inquiète davantage des causes politiques et sociales, de la décadence de la société et il définit la bourgeoisie comme une véritable «tumeur». Il n'est pas favorable non plus aux idées démocratiques, auxquelles il reproche d'avoir trompé le peuple en lui donnant l'impression d'avoir acquis des droits qui, en réalité, n'ont rien apporté de neuf dans la vie pratique de tous les jours. C'est donc le poète qui doit se charger du renouvellement de la société, en développant ses facultés pour les mettre au service de celle-ci en enclenchant les changements nécessaires pour un éveil social.

Le deuxième et dernier article signé par Kahn, dans «Le Symboliste», porte le titre Théâtres et il est publié en ouverture du quatrième numéro de la revue. Dans ce cas aussi le caractère politique est évident: selon Kahn, le théâtre a perdu sa fonction artistique pour n'être que le reflet des exigences de la classe bourgeoise dominante et, par conséquent, il s'est énormément appauvri. Il faut donc le renouveler à partir de sa forme: Kahn met en place ici des idées qui reviendront dans son «Théâtre de l'avenir» ${ }^{24}$. Il imagine des innovations essentielles: tout d'abord supprimer la récita-

(21) Les articles de Jean Moréas et de Paul Adam, nous l'avons déjà dit, sont publiés dans la première et la troisième sortie, tandis que ceux de Kahn se trouvent en ouverture de la deuxième et de la quatrième sortie.

(22) R. BiÉTRY, Les théories poétiques à l'époque symboliste (1883 - 1896), Genève, Slatkine, 2001, p. 111.

(23) Ibidem

(24) Cf. P. Durand, Le Théâtre du pauvre: de Mallarmé à Kahn et S. BASCH, Théâtre de l'avenir, mannequins du présent et nostalgie de guignol, dans 
tion, se concentrer sur la gestualité pour évoquer des sensations chez les spectateurs et, surtout, réintroduire la magnifique musique des grands maîtres tels que Wagner.

\section{Félix Fénéon}

Félix Fénéon est rédacteur en chef de «La Revue Indépendante» et collaborateur de «La Vogue» au moment où «Le Symboliste» voit le jour. Il participe à cet hebdomadaire en signant deux articles ${ }^{25}$. Le Musée du Luxembourg paru dans «Le Symboliste» du 15 octobre est un compte-rendu de la collection de peinture du Musée du Luxembourg auquel Fénéon reproche de ne pas avoir montré assez d'intérêt pour les peintres contemporains, ayant introduit des nouveautés thématiques, tels que Degas, Camille Pissarro, Edouard Manet, Rops, Puvis de Chavannes.

Les Illuminations d'Artbur Rimbaud dans «Le Symboliste» du 7 octobre propose un souvenir d'Arthur Rimbaud, dont on a, à l'époque, perdu les traces et dont les Illuminations viennent pourtant de paraître en volume ${ }^{26}$. Fénéon montre tout son enthousiasme pour une œuvre qu'il définit «hors de toute littérature et, probablement, supérieure à toute». Ainsi, par cet article, l'annexion de Rimbaud aux pères de l'école symboliste devient officielle.

\section{Plowert}

Dans les quatre numéros de la revue «Le Symboliste» nous trouvons la rubrique Parenthèses E Incidences; cette rubrique porte la signature «Plowert» et à ce sujet il est intéressant d'approfondir l'identité de notre mystérieux chroniqueur.

Jacques Plowert est un personnage de Les Demoiselles Goubert: moeurs de Paris, écrit par Jean Moréas et Paul Adam et publié en 1886. Ce personnage est un voyageur commercial, qui manque de son bras gauche, dont le nom a été repris pour créer un fantomatique collaborateur de notre hebdomadaire.

Dans le passé il y avait la tendance à croire que ce pseudonyme cachait tout simplement l'identité de Paul Adam, mais des études plus récentes, en particulier ceux d'Evanghélia Staed ${ }^{27}$, prouvent que plusieurs auteurs utilisaient ce nom de plume et que, en ce qui concerne notre rubrique, il s'agit bien «d'une rédaction à plusieurs» à laquelle participent, entre autres, sûrement Adam, Kahn, Fénéon et Moréas. Evanghélia Staed affirme que dans le premier numéro il est possible de distinguer cinq contributions différentes; dans le deuxième, deux; dans le troisième, cinq et dans le quatrième, trois.

La rubrique Parenthèses E Incidences est un espace consacré à la bataille verbale, où l'on peut contrebattre les critiques négatives et hasardées de la presse contre le journal même et ses collaborateurs.

Les rédacteurs du «Symboliste», protégés par un pseudonyme, décident, à travers cette petite rubrique collective, de répondre aux attaques multiples et de défendre leur identité en voulant prendre définitivement les distances des Décadents

Gustave Kabn (1859-1936), études réunies par S. BASCH, Paris, Classiques Garnier, 2009, pp. 1121 et pp. 23-43.

(25) Il faut préciser que, dans le premier numéro du «Symboliste», l'avis de la rédaction signale la participation de Fénéon et de Laforgue à la rubrique Des essais sur l'art, tandis qu'à partir du deuxième numéro, Fénéon a sa propre rubrique qui porte le titre Les cirques.

(26) Publication de «La Vogue».

(27) Cfr. E. Staed, Soixante et un mots de Gustave Kabn, recueillis par Jacques Plowert dans Gustave Kabn (1859-1936), études réunies par S. BASCH, cit., pp. 48-49. 
avec lesquels ils sont souvent confondus. Le langage qu'ils emploient dans ce contexte est souvent riche d'ironie et d'humour.

Dans cet espace on revient encore une fois sur Arthur Rimbaud, en essayant de donner quelques renseignements supplémentaires sur son errance. Il est bel et bien devenu désormais une figure mythique dont les œuvres seront de plus en plus recherchées et aimées ${ }^{28}$.

\section{Jules Laforgue}

Au moment où «Le Symboliste» est publié, Jules Laforgue vient de quitter son travail de lecteur auprès de l'impératrice allemande Augusta de Saxe-Weimar-Eisenach et de rencontrer une jeune anglaise qui deviendra sa femme ${ }^{29}$. La rédaction annonce trois «essais sur l'art» qui portent sa signature. Il s'agit en fait de deux textes de créations -où se mélangent la poésie et la prose- et d'une critique d'art: c'est ici qu'il fait paraître son morceau $\grave{A}$ propos d'Hamlet, qui suit la composition de la Moralite ${ }^{30}$, et $B o b o^{31}$, qui traite un sujet bien cher à l'auteur: l'inévitable opposition entre les sexes que l'on retrouve également dans le morceau sur la femme publié dans ses Mélanges posthumes $^{32}$. Cette réflexion rentre dans un cadre plus vaste qui conduit le poète à s'interroger sur le sens de la vie. Bien évidemment ses conclusions sont chargées d'un profond pessimisme accentué par l'influence qu'il reçoit des conceptions philosophiques de Schopenhauer et surtout de Hartmann ${ }^{33}$.

L'essai sur la peinture qui paraît dans le quatrième numéro du «Symboliste» est présent dans le recueil de critique d'art de l'auteur dans l'édition de Mireille Dottin: «Laforgue fait ici, - explique-t-elle - sous forme de flânerie "ça et là" un bilan humoristique de l'art en $1886 »^{34}$.

Il ne s'occupe ni de l'art impressionniste, comme l'avait fait Moréas dans son article basé sur le volume Les Impressionnistes en 1886 de Félix Fénéon, ni de la description d'un salon ou d'une exposition en particulier; expert d'art, ami du collectionneur Charles Ephrussi ${ }^{35}$, il se propose tout simplement d'exprimer librement son point de vue concernant des peintres et des œuvres de son époque qu'il a eues l'occasion d'admirer ${ }^{36}$.

(28) Dans la rubrique Parenthèses E Incidences du 22 octobre 1886, Plowert, sortant pour un instant des polémiques avec la presse, informe aussi ses lecteurs qu'il a été installé au Square Vintimille (devenu ensuite Square Berlioz) un monument pour rendre hommage au musicien Hector Berlioz, dix-sept ans après sa mort, et il relate la cérémonie officielle.

(29) En 1886 Jules Laforgue quitte son poste de lecteur qui recouvrait depuis 1881 et en même temps il rencontre la jeune anglaise Leah Lee qu'il épousera à Londres le 31 décembre; après le mariage il viendra s'établir à Paris jusqu'au 20 août 1887 , date de son décès.

(30) À propos d'Hamlet paraît dans «Le Symboliste» du 22 octobre 1886. Dans ce texte Laforgue mélange la prose avec quelques vers: des vers au sujet de la femme tirés du Concile féerique, publié pour la première fois dans «La Vogue» du 12 juillet 1886; ensuite il fait prononcer à Hamlet le vers «Aux armes, citoyens! Il n'y a plus de raison!» tiré du poème Simple agonie, paru pour la première fois dans «La Vogue» du 18 octobre 1886 et publié dans le recueil Derniers vers en 1890.

À ce propos cf. A. Holmès, Hamlet de Shakespeare au risque de Laforgue, «Vortex», n. 2, automne 2000, http://www.orsini.net/laforgue/vortex2/holmes 2 .htm.

(31) Dans l'article Bobo, paru dans «Le Symboliste» du 15 octobre, Laforgue introduit dans le texte une strophe où il exprime toujours sa pensée au sujet de la femme, cette strophe se retrouve, avec quelques variations, dans le poème L'aurore promise qui fait partie du recueil Des fleurs de bonne volonté, publié posthume en 1890.

(32) J. Laforgue Notes Sur la Femme dans Mélanges Posthumes, présentation de PHILIPPE BonNeFIS, Genève, Slatkine, 1979.

(33) Cfr. H. ScepI, Poétique de Jules Laforgue,

Paris, Presses Universitaires de France, 2000, p. 25.

(34) J. LAforgue Textes de critique d'art, réunis et présentés par Mireille DotTin, 1988, Presses Universitaires de Lille, p. 97.

(35) J. LAFORGUE Textes de critique d'art, réunis et présentés par M. DotTin, cit., p. 43.

(36) Dans son article, Laforgue précise que les 
Cet article aurait dû avoir une suite, ce qui est annoncé dans une petite note en bas de page, mais à cause de l'interruption brusque de la feuille elle ne sera jamais publiée.

\section{Jean Ajalbert}

Jean Ajalbert participe à la rédaction du «Symboliste» comme responsable de la rubrique Actualités; il signe trois articles et il offre aux lecteurs quelques-uns de ses vers extraits d'un volume en préparation ${ }^{37}$. Ses positions paraissent très proches de celle de Kahn: il vit dans une dimension plus politique. Ses idées paraissent pourtant au fil de l'heure, suivant les faits divers qui en sont l'occasion, elles parsèment justement les actualités. Dans Timbale milanaise, publié dans «Le Symboliste» du 7 octobre 1886, Ajalbert, à travers une longue métaphore, fait une critique à la société contemporaine, qu'il juge médiocre ${ }^{38}$. En particulier il dénonce l'attitude de la critique officielle par rapport à la nouvelle littérature et il revendique le droit à ne pas être compris par tout le monde comme quelque chose qui est depuis toujours spécifique de l'art. Comme pour Kahn, c'est la bourgeoisie, classe dominante ayant pris le pouvoir dans tous les domaines, même dans le domaine de l'art, qui est responsable de la perte de vitalité de la littérature, à laquelle le symbolisme se rebelle.

Dans Parallèles d'Horizontales («Le Symboliste» du 15 octobre 1886), dont le titre évoque celui du recueil de poèmes Les Horizontales d'Henri Beauclair qui date de 1885, Ajalbert annonce la mort de Musette, célèbre protagoniste de l'œuvre Scènes de la vie de bohème d'Henri Murger ${ }^{39}$. Ceci est prétexte d'une évocation nostalgique d'un temps désormais révolu, celui de la bohème, aussi bien que d'une réflexion sur la nouvelle attitude de l'artiste face à la société.

peintres vivent de modes, entre eux il faut distinguer les «lanceurs de modes» de ceux qu'il appelle «les obstinée des vieilles modes». Après cette réflexion l'auteur fait quelques considérations sur les tendances de son époque et il cite rapidement ceux qu'il définit les «Puvistes» (influencés par Puvis de Chavannes), James Whistler, Israëls, les «Fortunystes» (influencés par Maria Josep Bernat Fortuny Marsal), Mackart, Benjamin Constant, Fantin, Raffaëlli, Manet et Degas; après avoir présenté rapidement le panorama artistique de l'époque il annonce sa volonté de «flâner sans principes» et il commence à juger une série d'œuvres et de peintres comme Gustave Moreau, Puvis de Chavannes, Jules Breton, Auguste Pointelin, Antoine Guillemet, Alexandre Desgoffes, Fantin-Latour, Paul Flandrin, Carolus Duran.

(37) Il s'agit d'un extrait portant le titre $\mathrm{La}$ brume du soir publié dans «Le Symboliste» du 22 octobre 1886.

(38) Ajalbert introduit son article par la citation de la tragédie lyrique Mignon, mais il adopte tout de suite un style humoristique puisque il s'amuse à transformer en ton ludique les vers poétiques de cette tragédie: «Connais-tu le pays où fleurit l'oranger, où s'allongent d'inextricables macaronis, où s'échafaudent les cylindriques mortadelles? [...]».

Ce préambule, riche de stéréotypes à propos de l'Italie, est utile à Ajalbert pour introduire l'argument dont il veut parler au lecteur: «le jeûne de
Sueci» qui a pris le départ en Italie avant de répandre sa réputation à l'étranger.

Ce discours semble lié à une sorte d'expérience scientifique qui se proposait de découvrir un moyen infaillible pour améliorer la vie humaine et elle attribuait à la pratique du jeûne des propriétés miraculeuses. Ce thème permet à l'auteur d'établir une métaphore: le jeûne, qui est proposée comme un remède miraculeux, ne se limite pas à une privation physique, mais il dévient surtout une privation intellectuelle dans la société de son époque puisque la bourgeoisie, classe dominante, n'a pas la capacité de nourrir les esprits.

Il est intéressant de remarquer qu'Ajalbert s'amuse, tout le long de son article, à utiliser des jeux de mots: il emploie par exemple l'expression «Diète de Cologne» en jouant sur le double sens du mot «diète» qui désigne une assemblée en langage politique et une pratique alimentaire dans le domaine de la nutrition. Il utilise aussi le mot «sardines» qui se prête bien à la création d'ambiguïté puisqu'il désigne une espèce de poisson mais aussi les gallons cousus sur les manches des sous-officiers.

(39) Henri Murger (1822-1861) mène une vie de bohème qu'il décrit dans son œuvre principale Scènes de la vie de bohème (1847-1849). Cette œuvre, ainsi que son adaptation théâtrale La Vie de bohème, inspireront l'opéra La Bohème de Giacomo Puccini composée entre 1892 et 1895. 
Le dernier article d'Ajalbert, qui porte tout simplement le titre Actualités et qui paraît dans le dernier numéro de la revue, propose une vision d'ensemble de plusieurs événements qui semblent offrir un aperçu de la société de l'époque. L'auteur relate, entre autre, les luttes des travailleurs qui défendent leurs droits, ce qui est sans aucun doute un peu excentrique par rapport à l'esthétique de la revue ${ }^{40}$.

\section{Selwyn}

Au-delà des auteurs connus, «Le Symboliste» nous offre le mystère de quelques pseudonymes. L'un d'eux, Selwyn, signe dans le deuxième numéro de la revue un article critique qui porte le titre Louise Leclercq, de M. Paul Verlaine. Sous cette signature il est possible d'entrevoir un clin d'œil à Verlaine du point de vue de l'orientation sexuelle.

En effet, Georges Selwyn est un personnage du roman La Faustin d'Edmond de Goncourt publié en $1882^{41}$. Dans le roman, Selwyn, qui recouvre un rôle secondaire, est entouré d'une atmosphère d'homosexualité. Au moment où cette œuvre paraît, tout le monde croit reconnaître le poète anglais Swinburne dans le personnage de Georges Selwyn. Ainsi, «Georges Selwyn acquiert grâce à la fois à son référent réel, Swinburne, et au talent de Goncourt, une existence quasi réelle» ${ }^{42}$. Son article n'est pourtant que la présentation, sous forme d'éloge, de la première œuvre en prose, exception faite pour les Poètes Maudits, de Paul Verlaine, pour laquelle il identifie les lecteurs idéaux dans les «dilettanti de quintessence littéraire». Le sentiment d'une littérature aux marges de la société est donc évident: aussi bien que «Le Symboliste» est une «petite revue», les poètes chéris sont réservés à des amateurs, les seuls qui aient la sensibilité pour comprendre la nouvelle poésie.

\section{Charles Vignier, Gaston Dubreuilh et les extraits d'œuvres littéraires.}

Autour du «Symboliste» il y a aussi un certain nombre de collaborateurs chargés des comptes-rendus de théâtre (Charles Vignier) et de musique (Gaston Du-

(40) Dans ce même article Ajalbert fait aussi un petit compte rendu de la cérémonie officielle qui a marqué le départ des soixante-dix hôtes Cingalais qui avaient vécu pendant quelques semaines au Jardin zoologique d'acclimatation à Paris. À ce propos il faut savoir qu'à partir de 1877 et jusqu'en 1912 au Jardin zoologique d'acclimatation, on organisait des exhibitions ethnographiques. Pendant ces exhibitions des individus, provenant de pays lointains, étaient installés pendant quelques semaines dans la pelouse du jardin zoologique et ils étaient forcés de reproduire le même style de vie que celui du pays d'origine. Au début cette pratique était considérée intéressante pour permettre aux ethnographes de faire des études sans le besoin de se déplacer, mais avec le temps elle a fini par se transformer tout simplement en spectacle pour satisfaire le public curieux. La dernière exhibition ethnographique eut lieu en 1931 à Paris sur le site du Bois de Vincennes.

Pour terminer son article l'auteur annonce le décès de la Comtesse de la Fauconnière, une femme avec une vie assez singulière qui avait offert des arguments de conversation intéressants à la presse de l'époque puisqu'elle fut accusée de bigamie et ensuite «elle fut acquittée» par le tribunal. Cette femme, après son expérience, s'était intéressée d'une autre affaire de bigamie qui, à l'époque, avait été portée devant la justice: cette affaire concernait un tel Monsieur Lecouty qui vivait avec sa femme à Paris et qui avait eu l'occasion de connaître une charmante jeune femme d'Alfortville, Mademoiselle Levanneur.

Quelques temps après avoir fait connaissance de Mademoiselle Levanneur, Monsieur Lecouty la demanda en mariage et la cérémonie fut célébrée le 5 mars 1885. La première femme de Monsieur Lecouty, ayant des suspects au sujet de son époux, décida de prendre des renseignements et ainsi elle découvrit la vérité. Monsieur Lecouty fut accusé de bigamie et jugé par le tribunal d'Alfortville. Cfr. http://cgma.wordpress.com/2010/07/17/histoirede-maisons-viii-alfortville-depuis-1885/, site consulté le 4 janvier 2011.

(41) Encore une fois nos rédacteurs utilisent un pseudonyme emprunté au personnage d'un roman.

(42) www.frères-goncourt.fr/notices/3faustin.htm, site consulté le 25 janvier 2011. 
breuilh) $)^{43}$; un autre certain nombre publie dans la revue des extraits d'œuvres littéraires récentes ou encore inédites ${ }^{44}$.

En 1886 Charles Vignier occupe très souvent les pages de la presse non seulement en qualité de journaliste et écrivain mais aussi à cause d'événements personnels qui finiront pour devenir une partie intégrante de sa biographie.

En effet, suite à un article de journal qu'il avait publié dans «La Revue Moderniste» du $1^{\text {er }}$ février 1886 où il citait en passant une dispute entre l'écrivain Robert Caze et le critique Félicien Champsaur, il fut défié en duel par Robert Caze qui estimait avoir été outragé par les mots de cet article. Le duel eut lieu le 15 février 1886; Caze, qui avait entre autre choisi Paul Adam en qualité de témoin, fut blessé au foie et suite à cette blessure il mourut à la fin du mois suivant. Cet événement a fini pour lier indissolublement les noms des deux écrivains.

Mais l'année 1886 est aussi l'année où Charles Vignier publie son recueil de poèmes Centon et collabore à la rédaction du «Symboliste» ${ }^{45}$.

Dans le deuxième numéro de la revue nous retrouvons, en effet, son article Hamlet, qui se situe à l'intérieur du débat sur le théâtre, et qui montre à quel point Vignier subit, aussi bien que Laforgue, la séduction symbolique du prince danois. L'article est très intéressant car il est tout à fait réactionnaire: Vignier déclare préférer encore la version théâtrale proposée par Ducis, qui «garde son indépendance» et il refuse toutes les adaptations contemporaines. Il se montre convaincu qu'aussi le contenu de la tragédie de Shakespeare est trop révolu pour la sensibilité contemporaine et lorsqu'il veut donner une définition d'artiste, il choisit une représentation bien étrangère à Shakespeare, liée à la subjectivité: il considère un artiste «celui qui vient expliquer la féerie des contingences dans son moi» en utilisant tous les moyens qu'il a à sa disposition, c'est à dire «l'écriture, les couleurs, les signes musicaux et diverses substances pétrissables» pour créer son œuvre ${ }^{46}$.

Selon Vignier, il faut tirer Hamlet du côté de cette sensibilité: «Hamlet [...] est un homme de rêve, en proie à la vie, dans le temps» et il faut sans doute trouver un artiste sensible à ces caractéristiques pour aboutir à une représentation théâtrale digne de ce nom.

En conclusion de cette analyse, il est évident que «Le Symboliste» réussit à réunir sous l'apparence d'une esthétique analogue des positions qui paraissent à bien y voir tout à fait opposées.

(43) Gaston Dubreuilh signe l'article Courrier musical dans le troisième numéro du «Symboliste».

Dans cet article Dubreuilh définit la musique comme «la fusion d'une ligne mélodiques avec des harmonies»; malheureusement il relève un manque de sensibilité du public, même le plus expert, envers l'harmonie, qui est, comme il le dit, un élément constitutif essentiel de la musique. Ceci est la cause d'une compréhension toujours superficielle et incomplète des œuvres musicales. Dubreuilh est persuadé que le public arrive à bien comprendre seulement les manifestations d'«art extérieur» (comme par exemple la mélodie), qui donnent des sensations moins intenses mais qui ont l'avantage d'être plus immédiates donc facilement accessibles. L’harmonie au contraire nécessite d'une sensibilité bien plus profonde pour être comprise.

Dans l'article de Dubreuilh, caractérisé par l'emploi d'un langage très technique, nous pouvons remarquer l'influence exercée par Wagner.

(44) Dans «Le Symboliste» du 22 octobre 1886
Jean Ajalbert publie La brume du soir, un poème extrait d'un volume en préparation; Francis Poictevin publie deux extraits de son roman Seuls dans le numéro du 7 octobre et du 22 octobre; Paul Verlaine publie dans «Le Symboliste» du 15 octobre le poème Bouquet à Marie, ce poème changera par la suite son titre en Un conte et il sera publié dans le volume Amour en 1888. Maurice de Faramond publie dans «Le Symboliste» du 22 octobre le poème Vision extrait du volume Quintessences et, pour finir, Camille de Sainte Croix publie un extrait de son roman Contempler dans le dernier numéro de la revue.

(45) Le recueil de poèmes Centon de Charles Vignier est publié en 1886 par l'éditeur Léon Vanier.

(46) Vignier cite à ce sujet un article de Félix Fénéon paru dans le n. 10 du tome I de «La Vogue»: Fénéon affirmait qu'au nombre de ces diverses substances pétrissables «on doit ranger certains résidus de la saponification». 
Un exemple concret nous est offert, comme nous avons déjà eu l'occasion de le remarquer, par le choix de Moréas et Adam de publier leurs articles côte à côte, pour souligner leur rapport d'affinité, en prenant volontairement les distances de leur collègue Gustave Kahn qui, bien évidemment, exprime des idées fort différentes.

Cette division souligne l'existence d'une opposition assez nette à propos du concept de littérature et d'écrivain: d'un côté nous avons les partisans d'une littérature qui refuse toute dimension politique et sociale où le poète choisit de s'écarter de la foule vulgaire et de se concentrer uniquement sur l'acte de la création artistique. Parmi ceux-ci nous pouvons reconnaitre sans aucun doute Moréas et Adam.

D'autre côté nous avons des intellectuels tels que Kahn et, de manière plus nuancée, Ajalbert, qui conçoivent une littérature symboliste engagée du point de vue politique et social. En particulier, ils pensent que l'écrivain, au moyen de sa création, doit se charger de provoquer un éveil social capable de rétablir des valeurs humaines effacées par l'affirmation de la médiocrité de la classe bourgeoise.

D'ailleurs, comme le dit très clairement Kahn dans son article Difficulté de vivre, le but du «vivre ce serait [...] se développer en soi pour le bien de la collectivité»; le savant et surtout le poète, qui ont sans doute des capacités supérieures au commun de mortels, ont le devoir de «ramener l'inconscient au conscient, le conscient au divulgué» ${ }^{47}$. 